\title{
Síndrome de abstinencia neonatal debido a exposición prenatal al citalopram: a propósito de un caso
} Neonatal abstinence syndrome due to prenatally citalopram exposure: A case report

\author{
Dra. Sara Erol ${ }^{a}$ Dra. Beyza Ozcan ${ }^{a}$, Prof. Asoc. Dr. Istemi H. Celik ${ }^{a}$, Prof. Asoc. Dr. Ahmet Y. Bas ${ }^{a}$ y \\ Prof. Asoc. Dra. Nihal Demirel ${ }^{a}$
}

\begin{abstract}
RESUMEN
El síndrome de abstinencia neonatal (SAN) debido a la exposición prenatal al citalopram se desarrolla durante los primeros días de vida, incluso con una exposición al fármaco en dosis bajas. El tratamiento de apoyo es la primera opción, aunque puede usarse el fenobarbital en el tratamiento de este síndrome. No debe interrumpirse la lactancia. Debe hacerse un seguimiento de estos recién nacidos para establecer el desenlace del SAN y las consecuencias en el desarrollo neurológico. En este artículo presentamos el caso de un recién nacido con SAN debido a exposición al citalopram en una dosis más baja que lo informado previamente en la bibliografía durante los últimos seis meses del embarazo. Se utilizó el fenobarbital debido al fracaso del tratamiento no farmacológico.

Palabras clave: sindrome de abstinencia neonatal, citalopram, fenobarbital.
\end{abstract}

http:/ / dx.doi.org/10.5546/aap.2017.e424

Texto completo en inglés:

http: / / dx.doi.org/10.5546/ aap.2017.eng.e424

Cómo citar: Erol S, Ozcan B, Celik IH, et al. Síndrome de abstinencia neonatal debido a exposición prenatal al citalopram: a propósito de un caso. Arch Argent Pediatr 2017;115(6):e424-e427.

\section{INTRODUCCIÓN}

Los trastornos psiquiátricos, como la depresión mayor, la ansiedad y el trastorno obsesivo compulsivo, son frecuentes durante el embarazo y después de este. En un estudio de importancia realizado en los Estados Unidos se mostró una prevalencia del 13\% de trastornos de ansiedad y del estado de ánimo en las embarazadas o

a. Neonatología, Hospital Universitario y de Investigación en Ginecología Etlik Zubeyde Hanim, Angora, Turquía.

Correspondencia:

Dra. Sara Erol: sarasurmeli@gmail.com

Financiamiento: Ninguno.

Conflicto de intereses: Ninguno que declarar.

Recibido: 27-12-2016

Aceptado: 3-7-2017 puérperas. ${ }^{1}$ En general se usan antidepresivos para tratar estos trastornos. El tratamiento adecuado de la depresión durante el embarazo es de gran importancia para la salud materna, fetal y neonatal. ${ }^{2}$ El grupo de antidepresivos más recetado son los inhibidores selectivos de la recaptación de serotonina (ISRS), incluso en las embarazadas. $^{3}$

El uso de ISRS, en especial la paroxetina, la fluoxetina y la sertralina, durante el tercer trimestre del embarazo podría causar síndrome de abstinencia neonatal (SAN). Este síndrome también se denomina mala adaptación neonatal. ${ }^{4}$ Entre sus síntomas se incluyen nerviosismo, convulsiones, llanto anormal, trastornos alimentarios, dificultad respiratoria e hipoglucemia. ${ }^{5}$ En este artículo presentamos el caso de un recién nacido con SAN debido a exposición al citalopram durante los últimos seis meses del embarazo.

\section{A propósito de un caso}

Un bebé de sexo masculino nació por cesárea a las 36 semanas de gestación, con un peso de 3010 gramos, una longitud de $50 \mathrm{~cm}$ y un perímetro cefálico de $33 \mathrm{~cm}$. La madre tenía 40 años, había recibido atención prenatal adecuada y tenía antecedentes de depresión mayor. Había recibido citalopram $10 \mathrm{mg} /$ día desde los últimos dos trimestres del embarazo en adelante. Al nacer, los puntajes de Apgar del recién nacido fueron 8,9 y 10 en el minuto 1 , 5 y 10. Se hospitalizó al paciente en la unidad de cuidados intensivos neonatales debido a dificultad respiratoria en la primera hora después del nacimiento. Al ingreso tenía taquipnea con una frecuencia respiratoria de 68 respiraciones por minuto con tiraje subcostal e intercostal. El hemograma completo, la gasometría, la glucemia y los electrolitos séricos, incluida la concentración de calcio y magnesio, y la radiografía de tórax eran normales. El resultado de la proteína C-reactiva (PCR) en suero fue negativo. La dificultad respiratoria se trató con oxigenoterapia y mejoró gradualmente durante el primer día. 
A los 3 días de vida, el paciente presentó nerviosismo e hiperirritabilidad. Se le diagnosticó hipoglucemia ( $25 \mathrm{mg} / \mathrm{dl})$ e hipocalciemia (calcio ionizado: $3,1 \mathrm{mg} / \mathrm{dl}$ ). El nerviosismo y la hiperirritabilidad continuaron a pesar del tratamiento de reposición con glucosa y calcio por vía intravenosa. El tono muscular aumentó en pocas horas y comenzó a mostrar opistótonos de manera intermitente. Tenía hiperreactividad de los reflejos profundos y reflejo de Moro exagerado. Se administró la escala de puntuación modificada de Finnegan para detectar SAN. El puntaje fue 8 , correspondiente a SAN grave. Posteriormente, el neonatólogo revisó el puntaje del lactante cada dos horas. Se iniciaron los tratamientos no farmacológicos, como minimizar los estímulos ambientales, el reposo y el descanso adecuados, la lactancia y el método de envolver al bebé. Se inició la administración de fenobarbital $5 \mathrm{mg} / \mathrm{kg} /$ día, dividida cada 12 horas, porque tres puntajes consecutivos fueron $>8$ a pesar de los tratamientos no farmacológicos. Se realizaron una ecografía cerebral y un EEG para el diagnóstico diferencial; los resultados fueron normales. Después de tres días de tratamiento con fenobarbital, se redujeron el nerviosismo, la rigidez muscular y la hiperirritabilidad; la puntuación modificada de Finnegan era $<8$ y se disminuyó gradualmente la dosis de fenobarbital, la cual se discontinuó a los 14 días de vida. Los signos clínicos habían mejorado poco a poco hacia el día 10. El paciente recibió el alta el día 16 y su examen físico era normal.

\section{DISCUSIÓN}

Se ha demostrado la transferencia placentaria de ISRS de manera experimental en animales y en seres humanos. Estos fármacos no poseen metabolismo placentario. La transferencia placentaria del fármaco también se determina por la duración de la exposición al fármaco, la liposolubilidad, la fijación a las proteínas plasmáticas, el volumen de distribución y otros factores farmacocinéticos. ${ }^{6}$ Por definición, el síndrome de abstinencia o discontinuación de antidepresivos se produce en los primeros días después del cese repentino del fármaco, por ejemplo después del nacimiento, cuando la concentración del fármaco en el suero del recién nacido es indetectable o muy bajo como para producir un efecto biológico. Los síntomas más frecuentes incluyen irritabilidad, trastornos del sueño, llanto anormal, rigidez y temblores. ${ }^{7}$ El diagnóstico suele realizarse con base en los antecedentes prenatales, los hallazgos clínicos y la puntuación de Finnegan. La puntuación de Finnegan se desarrolló inicialmente para diagnosticar la abstinencia neonatal de opiáceos ${ }^{8}$ pero se ha empleado también para evaluar los síntomas neonatales en los lactantes expuestos a ISRS. ${ }^{9}$

En un estudio reciente se observó que la mayoría de los lactantes nacidos de madres que reciben tratamiento con ISRS durante embarazo estaban sanos durante el período neonatal. Se informó síndrome de abstinencia grave en el 3\% al $13 \%$ de estos estudios, mientras que la tasa de abstinencia leve fue del $22 \%$ debido a exposición materna a los ISRS. Los síntomas surgen principalmente en el sistema nervioso central. La hipoglucemia y los síntomas respiratorios fueron frecuentes..$^{10}$ Se informó síndrome de abstinencia neonatal debido a ISRS en 93 casos, incluidos seis casos con citalopram hasta 2003. ${ }^{11}$ En un estudio de cohorte retrospectivo más reciente llevado a cabo por Forsberg y col. se evaluó a 71 recién nacidos expuestos al citalopram durante el tercer trimestre con una posología mediana de $20 \mathrm{mg}$ / día (de $5 \mathrm{mg} /$ día a $80 \mathrm{mg} /$ día). ${ }^{10}$ Se diagnosticó abstinencia grave (puntuación de Finnegan $\geq 8$ ) y leve (puntuación de Finnegan= 4-7) tratada con terapia no farmacológica en 2 y 18 recién nacidos, respectivamente. Se observaron dificultad respiratoria e hipoglucemia en 5 y 8 recién nacidos, mientras que 10 de los recién nacidos fueron prematuros. No se halló ninguna correlación entre los ISRS y la dificultad respiratoria. Nordeng y col., informaron que hubo cinco lactantes con SAN debido a ISRS, incluido uno con exposición a citalopram. ${ }^{12}$ Se trató de un recién nacido de sexo masculino expuesto a citalopram, con una posología de $20 \mathrm{mg} /$ día a partir del quinto mes de embarazo que aumentó a $30 \mathrm{mg} /$ día dos meses antes del parto. No requirió tratamiento médico (puntuación de Finnegan $=4-6$ ) y recibió el alta a los 7 días de vida. No existe una dosis segura de citalopram para prevenir el SAN. La exposición a una dosis más baja que en estudios previos, como en nuestro paciente, que fue de $10 \mathrm{mg} / \mathrm{kg}$, podría provocar SAN. En este paciente, la dificultad respiratoria, usualmente presente con el uso de ISRS en el embarazo, se resolvió en 24 horas. La hipoglucemia y la hipocalciemia respondieron a la administración intravenosa de glucosa y calcio, como se informó en estudios previos. Dado que nuestro paciente era prematuro, el motivo de la hipoglucemia y la hipocalciemia podría 
ser la prematurez, no el SAN. Sin embargo, las manifestaciones neuromusculares se resolvieron tras el tratamiento con fenobarbital.

Se informaron concentraciones séricas de citalopram en los lactantes que representaban entre el $0,9 \%$ y el $4,3 \%$ de las concentraciones séricas de las madres entre dos semanas y dos meses después del parto. Según se ha informado, la ingesta de citalopram en los lactantes a través de la lactancia es del 0,7\% al 9\% de la dosis materna después de ajustar el peso y considerando una biodisponibilidad del citalopram del $100 \% .{ }^{13}$ A estos pacientes se les debe ofrecer la lactancia porque en estos estudios no se observaron efectos asociados al citalopram en los bebés durante la lactancia, mientras que se observó que la alimentación con leche maternizada era un factor de riesgo de SAN. ${ }^{4} \mathrm{La}$ determinación de las concentraciones plasmáticas de citalopram en suero de la madre y del recién nacido podría evitar los efectos secundarios del fármaco. Dada la extremadamente breve duración actual de la hospitalización de los recién nacidos y la vida media más prolongada de los ISRS, los síntomas podrían incluso aparecer después del alta. ${ }^{10}$ En nuestro hospital, los lactantes expuestos a antidepresivos permanecen hospitalizados al menos de 72 a 96 horas.

Son pocos los estudios realizados sobre los efectos a largo plazo de los ISRS administrados antes del nacimiento. La depresión materna es un factor de riesgo importante para el desarrollo de problemas con el desarrollo neurológico. ${ }^{14} \mathrm{Al}$ parecer no existen efectos importantes, pero es necesario realizar otros estudios.

El manejo de los recién nacidos incluye tratamientos farmacológicos y no farmacológicos. La terapia no farmacológica es la primera opción en todos los casos, pero tal vez no sea suficiente en los casos de abstinencia leve. Esta es fácilmente aceptable, menos costosa y menos controversial. La opción es intentar usar la terapia no farmacológica en todos los recién nacidos antes de iniciar el tratamiento farmacológico. El manejo adecuado abarca tratar al bebé delicadamente, alimentarlo a demanda y evitar atentamente despertarlo mientras duerme. En todos los recién nacidos, se deben implementar las prácticas de estimulación mínima continua con luz tenue y pocos ruidos. La alimentación frecuente, la leche maternizada con un alto contenido calórico y los alimentos espesados podrían cubrir las demandas nutricionales y metabólicas. ${ }^{15} \mathrm{El}$ fenobarbital es el fármaco de preferencia para el tratamiento del SAN causado por fármacos no opiáceos. El fenobarbital administrado en las dosis para la abstinencia no previene las convulsiones ni mejora los síntomas gastrointestinales. ${ }^{15}$ En nuestro paciente se observó una mejoría de los síntomas neurológicos.

A modo de conclusión, la mala adaptación neonatal y el SAN debido a la exposición prenatal a ISRS, como el citalopram, se desarrolla durante los primeros días de vida, incluso con una exposición al fármaco en dosis bajas. El tratamiento de apoyo es la primera opción, aunque puede usarse el fenobarbital en el tratamiento de este síndrome. Preferentemente debe continuarse con la lactancia. Debe hacerse un seguimiento de estos recién nacidos para establecer el desenlace del SAN y las consecuencias en el desarrollo neurológico.

\section{REFERENCIAS}

1. Vesga-Lopez O, Blanco C, Keyes K, et al. Psychiatric disorders in pregnant and postpartum women in the United States. Arch Gen Psychiatry 2008;65(7):805-15.

2. Patil AS, KullerJA, RheeEH. Antidepressants in pregnancy: a review of commonly prescribed medications. Obstet Gynecol Surv 2011;66(12):777-87.

3. Stephansson O, Granath F, Svensson T, et al. Drug use during pregnancy in Sweden - assessed by the Prescribed Drug Register and the Medical Birth Register. Clin Epidemiol 2011;3:43-50.

4. Kieviet N, van Ravenhorst M, Dolman KM, et al. Adapted Finnegan scoring list for observation of antidepressant exposed infants. J Matern Fetal Neonatal Med 2015;28(17):2010-4.

5. Reis M, Källén B. Delivery outcome after maternal use of antidepressant drugs in pregnancy: an update using Swedish data. Psychol Med 2010;40(10):1723-33.

6. Klinger G, Merlob P.SelectiveSerotonin Reuptake Inhibitor Induced Neonatal Abstinence Syndrome. Isr J Psychiatry Relat Sci 2008;45(2):107-13.

7. Koren G, Matsui D, Einarson A, et al. Is maternal use of selective serotonin reuptake inhibitors in the third trimester of pregnancy harmful to neonates? CMAJ 2005;172(11): 1457-9.

8. Finnegan LP, Connaughton JF, Kron RE Jr, et al. Neonatal abstinence syndrome: assessment and management. Addict Dis 1975;2(1-2):141-58.

9. Levinson-Castiel R, Merlob P, Linder N, et al. Neonatal abstinence syndrome after in utero exposure to selective serotonin reuptake inhibitors in term infants. Arch Pediatr Adolesc Med 2006;160(2):173-6.

10. Forsberg L, Navér L, Gustafsson LL, et al. Neonatal AdaptationinInfantsPrenatallyExposed to AntidepressantsClinical Monitoring Using Neonatal AbstinenceScore. PLoS One 2014;9(11):e111327.

11. Sanz EJ, De-las-Cuevas C, Kiuru A, et al. Selective serotonin reuptake inhibitors in pregnant women and neonatal withdrawal syndrome: a database analysis. Lancet 2005;365(9458):482-7.

12. Nordeng $H$, Lindemann $R$, Perminov KV, et al. Neonatal withdrawal syndrome after in utero exposure to selective serotonin reuptake inhibitors. Acta Paediatr 2001;90(3): 288-91. 
13. Franssen EJ, Meijs V, Ettaher F, et al. Citalopram serum and milk levels in mother and infant during lactation. Ther Drug Monit 2006;28(1):2-4.

14. Nulman I, Koren G, Rovet J, et al. Neurodevelopment of children following prenatal exposure to venlafaxine, selective serotonin reuptake inhibitors, or untreated maternal depression. Am J Psychiatry 2012;169(11):1165-74.

15. Kocherlakota P. Neonatal abstinence syndrome. Pediatrics 2014;134(2):e547-61. 